\title{
A modified INBC for FDTD analyzing of shielding cavity with thin conductive layers under plane wave incidence
}

\author{
Qing Si ${ }^{1}$, Zhengyu Huang ${ }^{1}$, Lihua Shi ${ }^{1 a)}$, Feng $\mathbf{L u}^{1,2}$, \\ Bin Chen ${ }^{1}$, and Wenwen Jiang ${ }^{1}$ \\ ${ }^{1}$ National Key Laboratory on Electromagnetic Environmental Effects and \\ Electro-optical Engineering, PLA University of Science and Technology, \\ Nanjing 210007, China \\ 2 Jiangsu Regulatory Bureau of Nuclear and Radiation Safety, \\ Nanjing 210019, P. R. China
}

a)shilhnj@163.com

\begin{abstract}
A modified impedance network boundary condition (INBC) is proposed for modeling electrically thin conductive layers (TCLs) under plane wave incidence in finite-difference time-domain (FDTD) method. Compared to the conventional INBC which represents the relationship between the electric and the magnetic fields collocated at two faces of the TCL, the modified INBC takes into consideration both the half-space-cell and half-time-step difference of the electric and the magnetic fields in Yee's grid. The primary advantage of the modified INBC is that it can be implemented in conventional FDTD algorithm without half-cell shift approximation. Numerical examples are presented to validate the efficiency and accuracy of the proposed method in analyzing thin layers with low conductivities.
\end{abstract}

Keywords: finite-difference time domain (FDTD) method, impedance network boundary condition (INBC), thin conductive layer (TCL)

Classification: Electromagnetic theory

\section{References}

[1] A. Taflove and S. C. Hagness: Computational Electrodynamics: The FiniteDifference Time-Domain Method (Artech House, Boston, MA 2000).

[2] M. Feliziani, F. Maradei and G. Tribellini: IEEE Trans. Electromagn. Compat. 41 (1999) 307. DOI:10.1109/15.809801

[3] J. Choo, C. Cho and J. Choo: IEICE Electron. Express 12 (2015) 20150361. DOI:10.1587/elex.12.20150361

[4] J. Inoue, T. Ogura, K. Hatanaka, K. Kintaka, K. Nishio, Y. Awatsuji and S. Ura: IEICE Electron. Express 10 (2013) 20130444. DOI:10.1587/elex.10.20130444 
[6] Xiaoli XI, Yongxing DU, Jiangfan LIU and Jinsheng ZHANG: IEICE Trans. Commun. E95-B (2012) 3895. DOI:10.1587/transcom.E95.B.3895

[7] M. S. Sarto: IEEE Trans. Electromagn. Compat. 41 (1999) 298. DOI:10.1109/ 15.809798

[8] T. Takenaka and T. Moriyama: IEICE Electron. Express 9 (2012) 1243. DOI:10.1587/elex.9.1243

[9] B. Gustavsen and A. Semlyen: IEEE Trans. Power Deliv. 14 (1999) 1052. DOI: $10.1109 / 61.772353$

[10] V. Nayyeri, M. Soleimani and O. M. Ramahi: IEEE Trans. Electromagn. Compat. 56 (2014) 385. DOI:10.1109/TEMC.2013.2286966

[11] R. Holland: IEEE Trans. Electromagn. Compat. 36 (1994) 32. DOI:10.1109/15. 265477

[12] R. B. Schulz, V. C. Plantz and D. R. Brush: IEEE Trans. Electromagn. Compat. 30 (1988) 187. DOI:10.1109/15.3297

\section{Introduction}

Thin conductive layer (TCL) is usually encountered in shielding structures for electromagnetic waves. To analyze the problem of wave penetration through TCLs with numerical computation methods such as the finite-difference time-domain (FDTD) method, the grid size must be very small in order to describe the fine structure with enough cells [1]. The computational burden when TCLs are modeled by fine grid becomes a big obstacle for three-dimensional analysis of a relatively large shielding structure.

To overcome this problem, the impedance network boundary conditions (INBCs) and other similar methods have been developed [2, 3, 4, 5, 6, 7, 8]. It uses equivalent transfer functions to describe the relationship of tangential field components on two surfaces of the TCL and the inner structure needs no longer to be modeled in FDTD. Therefore the cell size and the time step can be released to the normal requirement as those used in other parts of the analyzed reign.

Considering the frequency-dependent characteristics of INBC and its implementation in FDTD calculation, the method of iterative convolution is usually used to enhance the computation efficiency. A widely used general method is to approximate the surface impedance $\mathrm{Zs}$ and the transfer impedance $\mathrm{Zt}$ in INBC by series of s-domain rational functions using vector-fitting method [9]. These rational functions in frequency domain are then converted to their equivalent time-domain formulations by recursive convolution [2,7].

An important problem affecting the application of INBC in FDTD is the inconsistency of magnetic field nodes in INBC with those used in Yee's grid. For example, the surface impedance $\mathrm{Zs}$ describes the relationship between the tangential $\mathrm{E}$ filed and the tangential $\mathrm{H}$ field at the same point of the TCL surface. However, in Yee's grid E and H cannot be collocated and there should be a halfspace-step and a half-time-step difference in FDTD calculations. In practical operations the computed $\mathrm{H}$ field is shifted by a half space step and a half time step to fit the collocation requirement of INBC, which makes a compromise between the computational accuracy and the feasibility of using INBC in FDTD. To solve the 'H-node shift' problem, Nayyeri et al. proposed a new formulation of 
INBC in FDTD [10], where a separate difference grid in vicinity to TCL surfaces is established and a set of simultaneous equations is designed to solve the time-shift problem in $\mathrm{H}$ field.

As discussed above, the essence of ' $\mathrm{H}$-node shift' problem is the in-consistency of INBC field nods with Yee's grid. Previous researches focus mainly on the modification of FDTD to fit INBC's requirement, while in this paper we propose an alternative approach to fit the FDTD's requirement by modifying the formulation of INBC. In our modified method, two cascaded networks are added to consider the half-space-cell shift of magnetic field in the whole INBC network, and the exponential time difference (ETD) formulation [11] is applied to implement the modified INBC in FDTD calculation to keep accuracy. The modified INBC can describe the features of TCL directly by E and H fields on Yee's grids. The primary advantage is that it can be implemented in the conventional FDTD method without the half-grid approximation. Numerical examples are presented to validate the efficiency and accuracy of the proposed method. Results show that the modified INBC has higher accuracy for TCLs. The results for low-conductivity cases will be presented as an emphasis in the following parts.

\section{Formulations}

For an uniform TCL with thickness $d$ and illuminated by an oblique plane wave with oblique incidence at angle $\theta$, the field components used in FDTD and INBC are shown in Fig. 1 , where $E_{\mathrm{xa}}, E_{\mathrm{ya}}, H_{\mathrm{xa}}, H_{\mathrm{ya}}$ and $E_{\mathrm{xb}}, E_{\mathrm{yb}}, H_{\mathrm{xb}}, H_{\mathrm{yb}}$ are the components of the electric and magnetic fields tangential to the external faces of the TCL. We use subscribes $a-1 / 2$ and $b+1 / 2$ to represent the computational grids at half-space-step before and behind the TCL. Suppose the unit vector of incident wave is

$$
\hat{a}_{z}=\sin \theta \hat{y}+\cos \theta \hat{z}
$$

the oblique incident wave can be decomposed into the vertically polarized wave and the parallel polarized wave. According to the transmission line network theory, the characteristic impedance of transmission line is the ratio between the tangential electric field and tangential magnetic field, and the equivalent propagation constant

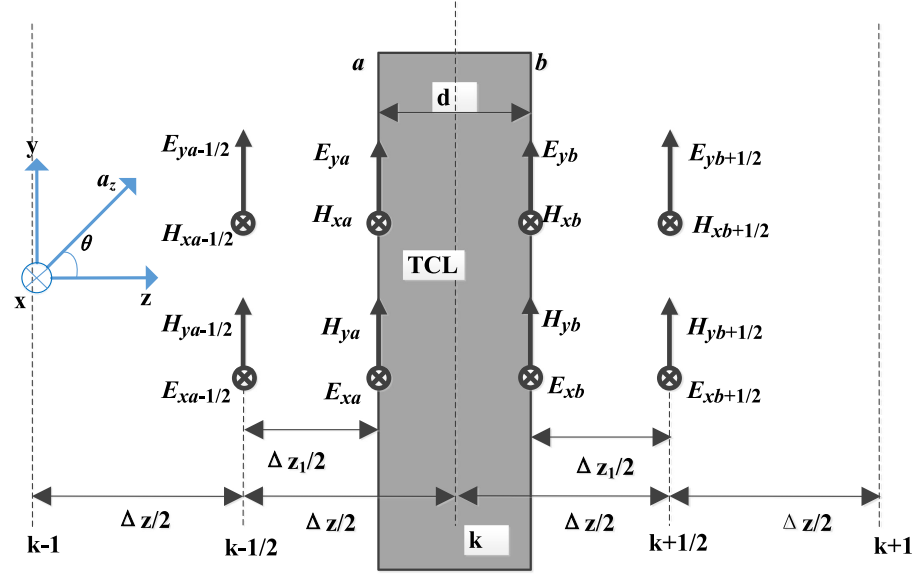

Fig. 1. Tangential electric and magnetic fields used in INBC of TCL 
in the conductor is the z-direction projection of the propagation constant of the refracted wave. In the next part, we will take the parallel polarized wave as an example to deduce the modified INBC.

In free space, the characteristic impedance of parallel polarized wave is $\eta_{/ /}=$ $\eta_{0} \cos \theta$, where $\eta_{0}=\sqrt{\mu_{0} / \varepsilon_{0}}$. In the TCL, the propagation constant is $\gamma_{1}=\gamma \cos \theta_{1}$ where $\gamma=\sqrt{j \omega \mu(\sigma+j \omega \varepsilon)}$ and the refraction angle $\theta_{1}$ can be written according to Snell's law

$$
\sin \theta_{1}=\sin \theta \sqrt{\varepsilon_{0} / \varepsilon}
$$

where $\varepsilon$ is the complex permittivity in TCL

$$
\varepsilon=\varepsilon_{0} \varepsilon_{r}\left(1+\frac{\sigma}{j \omega \varepsilon_{0} \varepsilon_{r}}\right)
$$

The characteristic impedance with parallel polarized wave is $\eta_{1 / /}=\eta \cos \theta_{1}$, where $\eta=\sqrt{j \omega \mu /(\sigma+j \omega \varepsilon)} . \mu$ and $\sigma$ are the permeability and electrical conductivity of the TCL, respectively.

The relationship between $\left(E_{\mathrm{ya}-1 / 2}, E_{\mathrm{yb}-1 / 2}\right)$ and $\left(H_{\mathrm{xa}-1 / 2}, H_{\mathrm{xb}+1 / 2}\right)$ in frequency domain can be expressed in the following matrix forms:

$$
\begin{gathered}
{\left[\begin{array}{c}
-E_{y a-1 / 2} \\
H_{x a-1 / 2}
\end{array}\right]=\Phi_{/ /}\left[\begin{array}{c}
-E_{y b+1 / 2} \\
H_{x b+1 / 2}
\end{array}\right]=\left[\begin{array}{ll}
\Phi_{11 / /} & \Phi_{12 / /} \\
\Phi_{21 / /} & \Phi_{22 / /}
\end{array}\right]\left[\begin{array}{c}
-E_{y b+1 / 2} \\
H_{x b+1 / 2}
\end{array}\right]} \\
{\left[\begin{array}{c}
-E_{y a-1 / 2} \\
H_{x a-1 / 2}
\end{array}\right]=A_{/ /}\left[\begin{array}{c}
-E_{y a} \\
H_{x a}
\end{array}\right]} \\
{\left[\begin{array}{c}
-E_{y b} \\
H_{x b}
\end{array}\right]=A_{/ /}\left[\begin{array}{c}
-E_{y b+1 / 2} \\
H_{x b+1 / 2}
\end{array}\right]}
\end{gathered}
$$

where

$$
\begin{gathered}
\Phi_{/ /}=A_{/ /} B_{/ /} A_{/ /} \\
A_{/ /}=\left[\begin{array}{cc}
\cos \beta\left(\Delta z_{1} / 2\right) & j \eta_{/ /} \sin \beta\left(\Delta z_{1} / 2\right) \\
j \eta_{/ /}^{-1} \sin \beta\left(\Delta z_{1} / 2\right) & \cos \beta\left(\Delta z_{1} / 2\right)
\end{array}\right]=\left[\begin{array}{cc}
a_{11 / /} & a_{12 / /} \\
a_{21 / /} & a_{22 / /}
\end{array}\right] \\
B_{/ /}=\left[\begin{array}{cc}
\cosh \left(\gamma_{1} d\right) & \eta_{1 / /} \sinh \left(\gamma_{1} d\right) \\
\eta_{1 / /}^{-1} \sinh \left(\gamma_{1} d\right) & \cosh \left(\gamma_{1} d\right)
\end{array}\right] \\
\beta=\omega \sqrt{\mu_{0} \varepsilon_{0}} \cos \theta
\end{gathered}
$$

Using matrix equation (4) (6) we can obtain the relationship between the tangential electric field components on the surfaces $\left(E_{\mathrm{ya}}, E_{\mathrm{yb}}\right)$ and the tangential magnetic field components in the nodes located respectively at half-space-step before and behind the TCL surfaces $\left(H_{\mathrm{xa}-1 / 2}, H_{\mathrm{xb}+1 / 2}\right)$. In other words, we can deduce a new INBC in accordance with the Yee's FDTD grid. Taking the derivation from $\left[H_{\mathrm{xa}-1 / 2} E_{\mathrm{xb}+1 / 2}\right]^{\mathrm{T}}$ to $\left[E_{\mathrm{ya}} E_{\mathrm{yb}}\right]^{\mathrm{T}}$ we have following equations from (4)

$$
\begin{gathered}
E_{y a-1 / 2}=-Z_{0 / /} H_{x a-1 / 2}+Z_{t / /} H_{x b+1 / 2} \\
E_{y b+1 / 2}=Z_{d / /} H_{x b+1 / 2}-Z_{t / /} H_{x a-1 / 2}
\end{gathered}
$$

where 


$$
Z_{0 / /}=\Phi_{11 / /} / \Phi_{21 / /}, Z_{t / /}=1 / \Phi_{21 / /}, Z_{d / /}=\Phi_{22 / /} / \Phi_{21 / /}
$$

Applying reciprocity condition to (5), we can obtain

$$
E_{y a-1 / 2}=\left(1 / a_{22 / /}\right) E_{y a}-\left(a_{12 / /} / a_{22 / /}\right) H_{x a-1 / 2}
$$

and substituting (14) into (11), we have

$$
E_{y a}=Z_{11 / /} H_{x a-1 / 2}+Z_{12 / /} H_{x b+1 / 2}
$$

where

$$
Z_{11 / /}=\left(a_{12 / /}-a_{22 / /} Z_{0 / /}\right), Z_{12 / /}=a_{22 / /} Z_{t / /}
$$

By inserting (6) into (12), we get

$$
E_{y b / /}=Z_{21 / /} H_{x a-1 / 2}+Z_{22 / /} H_{x b+1 / 2}
$$

where

$$
Z_{21 / /}=\left(a_{11 / /} Z_{d / /}-a_{12 / /}\right), Z_{22 / /}=-a_{22 / /} Z_{t / /}=-Z_{12 / /}
$$

Now we have obtained a new set of modified INBCs formulations described as (15) and (17). And the case of vertically polarized wave is similar to the case of parallel polarized wave above, notice that the characteristic impedance in free space and in the TCL is $\eta_{\perp}=\eta_{0} / \cos \theta$ and $\eta_{1 \perp}=\eta / \cos \theta_{1}$ respectively. The relationship between $E_{x}$ and $H_{y}$ in frequency domain can be expressed in the following matrix forms

$$
\begin{aligned}
& {\left[\begin{array}{c}
E_{x a-1 / 2} \\
H_{y a-1 / 2}
\end{array}\right]=\Phi_{\perp}\left[\begin{array}{l}
E_{x b+1 / 2} \\
H_{y b+1 / 2}
\end{array}\right]=\left[\begin{array}{ll}
\Phi_{11 \perp} & \Phi_{12 \perp} \\
\Phi_{21 \perp} & \Phi_{22 \perp}
\end{array}\right]\left[\begin{array}{l}
E_{x b+1 / 2} \\
H_{y b+1 / 2}
\end{array}\right] } \\
& {\left[\begin{array}{l}
E_{x a-1 / 2} \\
H_{y a-1 / 2}
\end{array}\right]=A_{\perp}\left[\begin{array}{c}
E_{x a} \\
H_{y a}
\end{array}\right] } \\
& {\left[\begin{array}{l}
E_{x b} \\
H_{y b}
\end{array}\right]=A_{\perp}\left[\begin{array}{l}
E_{x b+1 / 2} \\
H_{y b+1 / 2}
\end{array}\right] }
\end{aligned}
$$

where

$$
\begin{gathered}
\Phi_{\perp}=A_{\perp} B_{\perp} A_{\perp} \\
A_{\perp}=\left[\begin{array}{cc}
\cos \beta\left(\Delta z_{1} / 2\right) & j \eta_{\perp} \sin \beta\left(\Delta z_{1} / 2\right) \\
j \eta_{\perp}^{-1} \sin \beta\left(\Delta z_{1} / 2\right) & \cos \beta\left(\Delta z_{1} / 2\right)
\end{array}\right]=\left[\begin{array}{cc}
a_{11 \perp} & a_{12 \perp} \\
a_{21 \perp} & a_{22 \perp}
\end{array}\right] \\
B_{\perp}=\left[\begin{array}{cc}
\cosh \left(\gamma_{1} d\right) & \eta_{1 \perp} \sinh \left(\gamma_{1} d\right) \\
\eta_{1 \perp}^{-1} \sinh \left(\gamma_{1} d\right) & \cosh \left(\gamma_{1} d\right)
\end{array}\right]
\end{gathered}
$$

By analyzing the simultaneous equations (19)-(21) we can obtain

$$
\begin{gathered}
E_{x a-1 / 2}=Z_{0 \perp} H_{y a-1 / 2}-Z_{t \perp} H_{y b+1 / 2} \\
E_{x b+1 / 2}=-Z_{d \perp} H_{y b+1 / 2}+Z_{t \perp} H_{y a-1 / 2}
\end{gathered}
$$

where

$$
Z_{0 \perp}=\Phi_{11 \perp} / \Phi_{21 \perp}, Z_{t \perp}=1 / \Phi_{21 \perp}, Z_{d \perp}=\Phi_{22 \perp} / \Phi_{21 \perp}
$$

Similarly we can get 


$$
\begin{aligned}
& E_{x a}=Z_{11 \perp} H_{y a-1 / 2}+Z_{12 \perp} H_{y b+1 / 2} \\
& E_{x b}=Z_{21 \perp} H_{y a-1 / 2}+Z_{22 \perp} H_{y b+1 / 2}
\end{aligned}
$$

where

$$
\begin{gathered}
Z_{11 \perp}=a_{22 \perp} Z_{0 \perp}-a_{12 \perp}, Z_{12 \perp}=-a_{22 \perp} Z_{t \perp} \\
Z_{21 \perp}=a_{11 \perp} Z_{t \perp}, Z_{22 \perp}=a_{12 \perp}-a_{11 \perp} Z_{d \perp}
\end{gathered}
$$

Compared with the conventional INBCs, which describe the relationship between the tangential EM fields on two surfaces of TCL, the modified INBC considers the relationship of EM fields on the TCL surface with EM fields at halfgrid nodes outside the surface. In the modified INBC, $H_{x a-1 / 2}$ and $H_{x b+1 / 2}$ are all on the nodes located at Yee's FDTD difference meshes. Thus the updating equations for these components will be the same as those used in the conventional FDTD method. Besides, this method just provides the probability to calculate the thin structure by sparse grid, the Courant's stability condition cannot be violated.

In FDTD calculation, the modified INBC needs also to be transformed into their time-domain representations. Similar to the method adopted in [2] and [7], the $Z_{\mathrm{ij} / /}$ and $\mathrm{Z}_{\mathrm{ij} \perp}$ are expressed as a set of rational polynomials of complex frequency $\mathrm{j} \omega$ by using vector fitting [9], and then transformed into time-domain difference equations. We will take $\mathrm{Z}_{\mathrm{ij} / /}$ as an example to illustrate the method in the following parts.

In (15), two frequency-dependent impedance parameters $Z_{11 / /}(\omega)$ and $Z_{12 / /}(\omega)$ can be approximated with a set of first-order rational fractions expressed as

$$
Z_{11 / /}(\omega)=\sum_{m=1}^{m_{a}} \frac{r_{1 m / /}}{j \omega-p_{1 m / /}}, Z_{12 / /}(\omega)=\sum_{m=1}^{m_{b}} \frac{r_{2 m / /}}{j \omega-p_{2 m / /}}
$$

We use two instrumental variables to represent the first and the second scheme in (15)

$$
E_{y a}(\omega)=\sum_{m=1}^{m_{a}} G_{1 m / /}(\omega)+\sum_{m=1}^{m_{b}} G_{2 m / /}(\omega)
$$

where

$$
\begin{aligned}
G_{1 m / /}(\omega) & =\frac{r_{1 m / /}}{j \omega-p_{1 m / /}} H_{x a-1 / 2}(\omega) \\
G_{2 m / /}(\omega) & =\frac{r_{2 m / /}}{j \omega-p_{2 m / /}} H_{x b+1 / 2}(\omega)
\end{aligned}
$$

In time domain, (34) and (35) become

$$
\begin{aligned}
& \frac{\partial G_{1 m / /}(t)}{\partial t}-p_{1 m / /} G_{1 m / /}(t)=r_{1 m / /} H_{x a-1 / 2}(t) \\
& \frac{\partial G_{2 m / /}(t)}{\partial t}-p_{2 m / /} G_{2 m / /}(t)=r_{2 m / /} H_{x b+1 / 2}(t)
\end{aligned}
$$

Approximated with ETD [11] in discrete time,

$$
\begin{aligned}
& G_{1 m / /}^{n+1}=e^{p_{1 m / /} \Delta t} G_{1 m / /}^{n}+\left(1-e^{p_{1 m / /} \Delta t}\right) \frac{r_{1 m / /}}{-p_{1 m / /}} H_{x a-1 / 2}^{n+1 / 2} \\
& G_{2 m / /}^{n+1}=e^{p_{2 m / /} \Delta t} G_{2 m / /}^{n}+\left(1-e^{p_{2 m / /} \Delta t}\right) \frac{r_{2 m / /}}{-p_{2 m / /}} H_{x b+1 / 2}^{n+1 / 2}
\end{aligned}
$$

where $\Delta t$ is the sample interval. 
In analyzing TCL with 3-D FDTD, the only modification of conventional operation is to substitute the updating equation of FDTD at surfaces of TCL by the following equation

$$
\begin{aligned}
E_{y}^{n+1}(i, j+1 / 2, a)= & \sum_{m=1}^{m_{a}} G_{1 m / /}^{n+1}(i, j+1 / 2, a) \\
& +\sum_{m=1}^{m_{b}} G_{2 m / /}^{n+1}(i, j+1 / 2, a)
\end{aligned}
$$

where

$$
\begin{aligned}
& G_{1 m / /}^{n+1}(i, j+1 / 2, a)=e^{p_{1 m / /} \Delta t} G_{1 m / /}^{n}(i, j+1 / 2, a) \\
& \quad+\left(1-e^{p_{1 m / /} \Delta t}\right) \frac{r_{1 m / /}}{-p_{1 m / /}} H_{x}^{n+1 / 2}(i, j+1 / 2, a-1 / 2) \\
& G_{2 m / /}^{n+1}(i, j+1 / 2, a)=e^{p_{2 m / /} \Delta t} G_{2 m / /}^{n}(i, j+1 / 2, a) \\
& \quad+\left(1-e^{p_{2 m / /} \Delta t}\right) \frac{r_{2 m / /}}{-p_{2 m / /}} H_{x}^{n+1 / 2}(i, j+1 / 2, b+1 / 2)
\end{aligned}
$$

The realization of (17) can all be handled similarly as the above-mentioned approach for (15).

\section{Numerical demonstrations}

In this section, two examples are presented to demonstrate the efficiency of the proposed method.

\subsection{1-D example}

To validate the accuracy and stability of the modified INBC in the case of low conductivities besides the case of high conductivities, an infinite conductive planar illuminated by a TEM plane wave is analyzed. The planar is a single-layered structure with the thickness of $1 \mathrm{~mm}$ and in an assumption that it is perpendicular to $z$-direction. The space grid is kept as $\Delta z=50 \mathrm{~mm}$, which is much bigger than the thickness of the planar and the time step is chosen as $\Delta t=\Delta z / c \approx 0.166 \mathrm{~ns}$ where $c$ is the speed of light in the air, which satisfied with the Courant's stability condition. A Gaussian pulse is used as the incident plane wave profile: $g(t)=$ $\exp \left[-\left(t-t_{0}\right)^{2} / \tau^{2}\right]$, where $\tau=n_{c} \Delta \mathrm{z} /(2 \mathrm{c}), t_{0}=4.5 \tau$ and $n_{c}=48$ is the ratio of wavelength to grid size. Recording point of penetrated electric field is put at the distance of $200 \mathrm{~mm}$ ( 4 cells) from the back of planar/air interface.

For the case of the slab with conductivity $\sigma=100 \mathrm{~S} / \mathrm{m}$ and relative permittivity $\varepsilon_{r}=2$, a comparison between the electric fields calculated by the analytical method [12], the original INBC-FDTD method and the modified INBC-FDTD method is investigated. For the two INBC methods, the highest frequency $f_{\max }=125 \mathrm{MHz}$ of the incident fields is set to perform the vector fitting process with 20 orders model. And the analytical method is used to obtain the reference result to INBC methods. Fig. 2 shows the relative error to the analytical method in frequency domain, it can be found that the modified INBC gives much higher accuracy both in amplitude and phase. The relative error $R$ is calculated as follows: 


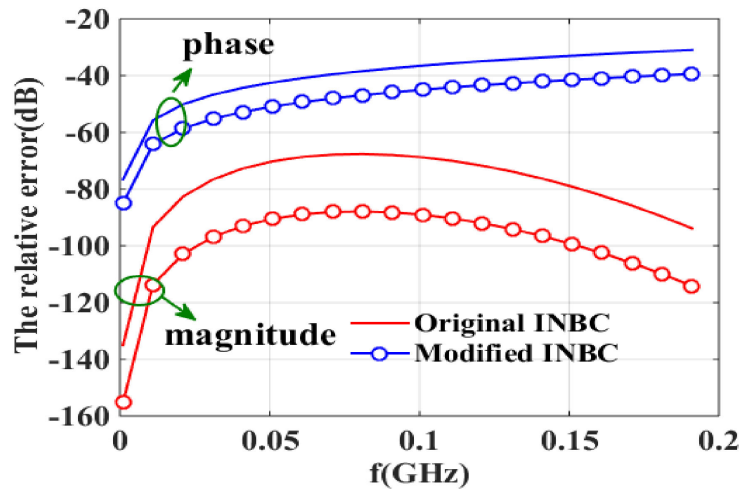

Fig. 2. Relative errors of the two methods in frequency domain

$$
R=20 \lg \left(\left|Y_{\text {Analytical }}(f)-Y_{I N B C}(f)\right| /\left[\max \left(\left|Y_{\text {Analytical }}(f)\right|\right)\right]\right)
$$

where $Y$ represents amplitude or phase. The result of INBC-FDTD is transformed into frequency domain by fast Fourier transformation. It is clear that the error induced by 'H-node shift' in the original INBC is reduced in the modified INBC. Fig. 3 shows the mean relative error (MRE) with different mesh sizes in frequency domain. The MRE is calculated as follows:

$$
\operatorname{MRE}=\left(\sum_{i=1}^{N} R\left(f_{i}\right)\right) / N
$$

where $N$ is the total numbers of frequency points, $R\left(f_{i}\right)$ is the relative error shown in (43) for each frequency point $f_{i}$. The MRE will become higher as the mesh size increases for both of the two methods, but the modified method keeps less calculation errors under different mesh size.

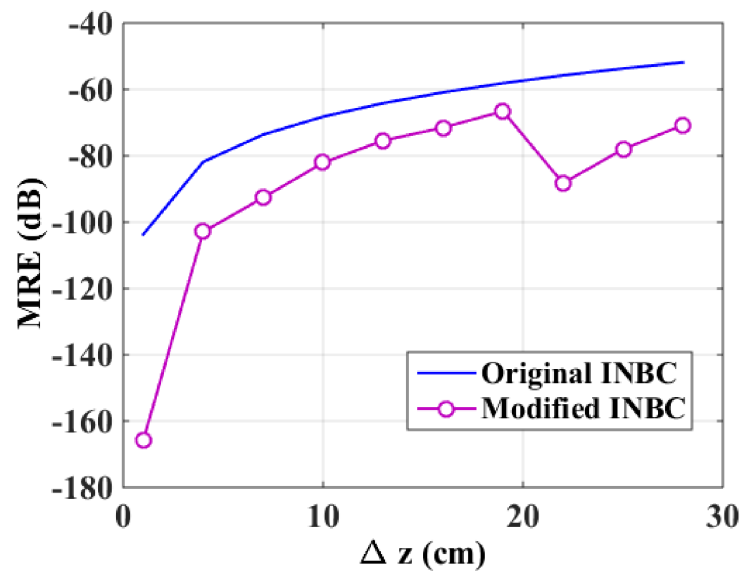

Fig. 3. The relative errors when space step varies

For lower conductivity, the proposed INBC can also keep good accuracy and stability. Fig. 4 shows that when the conductivity reduces to $1 \mathrm{~S} / \mathrm{m}$ and other conditions keep the same as previous case, the results in frequency domain by modified INBC are agreeable to the analytical results. 

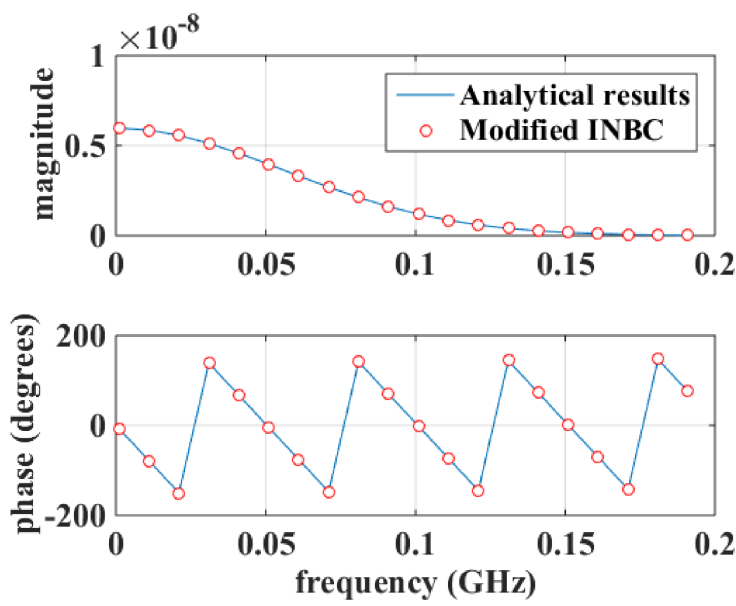

Fig. 4. The frequency domain results for $\sigma=1 \mathrm{~S} / \mathrm{m}$

\subsection{3-D example}

A $1 \mathrm{~m} \times 1 \mathrm{~m} \times 1 \mathrm{~m}$ cubic cavity shown in Fig. 5 with a $1 \mathrm{~mm}$ TCL on its bottom surface is analyzed. The other five surfaces of the cavity are set as perfect conductor. The plane wave with oblique incidence penetrated through the bottom surface into the cavity. Almost all the parameters of the incident wave is the same as before in 3.1 1-D example but the incident angle is $60^{\circ}$ and the polarizing angle of electric field is $14^{\circ}$. To model this 3-D structure, it needs tremendous memory consumptions and long simulation time for conventional FDTD if the grid size is chosen to fit the $1 \mathrm{~mm}$ TCL. Even if adopting the gradient mesh method, the calculation time is also unacceptable since the least time interval is determined by the least grid size. To further validate the proposed INBC, penetrated electric field at the center point of the cavity is recorded and compared with the solution from the commercial CST studio. In CST, the highest frequency is $400 \mathrm{MHz}$, the lines per wavelength is 10 , the minimum gird size is $0.2 \mathrm{~mm}$ for TCL and the maximum size is $15 \mathrm{~mm}$ for the other parts of the analyzed region. While in INBC-FDTD, the cell size is $50 \mathrm{~mm}$.

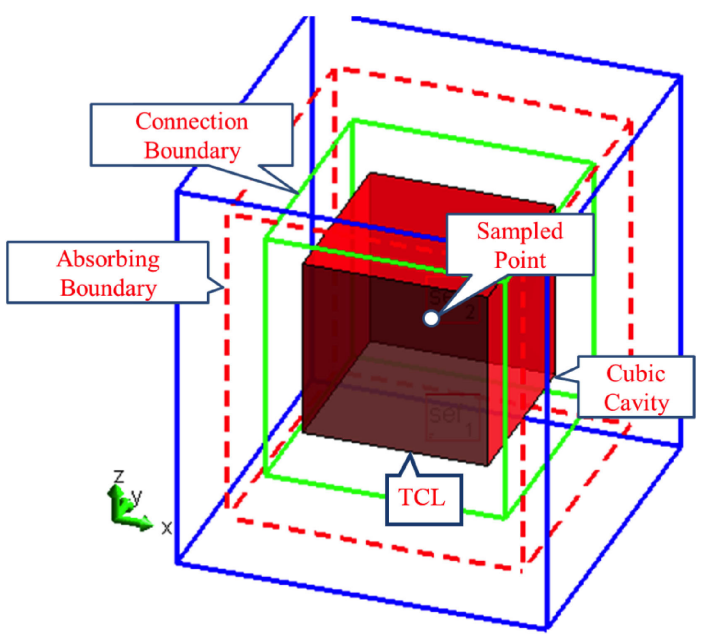


Fig. 6 and Fig. 7 show the recorded electric field $E_{x}$ and $E_{y}$ for the modified INBC are agreeable to CST's result when the conductivity of TCL is $100 \mathrm{~S} / \mathrm{m}$, which means the modified INBC method also behaves well in the low conductivity case for 3-D structures. The CPU time of the modified INBC-FDTD is about 3.1 minutes, which is far less than $8973 \mathrm{~s}$ of CST.

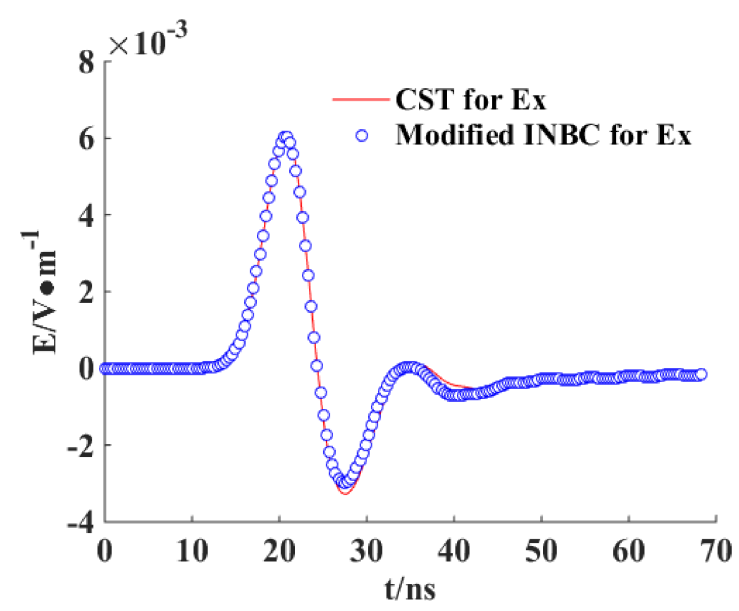

Fig. 6. The results of Ex for CST and modified INBC method

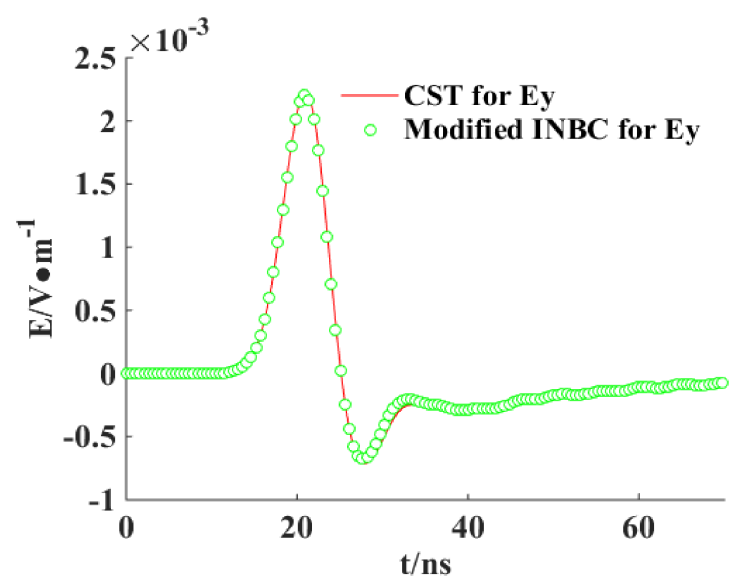

Fig. 7. The results of Ey for CST and modified INBC method

In fact if $\varepsilon \gg \varepsilon_{0}$ in (2), the refraction angle $\theta_{1}$ can be considered as $0^{\circ}$, which means the method of dealing with oblique incidence can be simplified to normal incidence. For example, suppose $\varepsilon_{\mathrm{r}}=1$ and $\mu_{0}=1$, the definition of good conductor is

$$
\frac{\sigma}{\omega \varepsilon_{r} \varepsilon_{0}} \geq 10
$$

If the frequency is $125 \mathrm{MHz}$, the critical value of $\sigma$ is $6.9 \times 10^{-2} \mathrm{~S} / \mathrm{m}$. So for most of conductors, propagation of EM waves in TCLs can be regarded as in the 


\section{Conclusions}

A modified INBC analyzing TCLs efficiently and accurately is deduced. Compared with the original INBC, which uses tangential field components at two surfaces of the TCL, the modified INBC gives the relationship of the tangential electric field with the tangential magnetic field at half-grid nodes from the TCL surface. This modification allows the field components in INBC to locate exactly at Yee's grid in FDTD. Therefore the approximation of the magnetic field on TCL surfaces is avoided in INBC-FDTD calculation. Both 1-D examples for normal incidence and 3-D example for oblique incidence have verified the accuracy of the proposed method. The formulation of the modified INBC under oblique incidence in Section 2 can be further simplified using $\theta=0^{\circ}$. 